REVUE HYBRIDE DE L’ÉDUCATION

Conseillers pédagogiques (CP) du primaire au cégep

\title{
Portraits de pratiques de deux CP collaboratifs
}

Auteur(s)

Nathalie MURRAY, Ph. D., conseillère pédagogique, Cégep de Jonquière, Canada

nathaliemurray@cegepjonquiere.ca

Étienne PERRON, conseiller pédagogique, Commission scolaire De La Jonquière, Canada

etienne.perron@csjonquiere.qc.ca 


\section{REVUE HYBRIDE DE L'ÉDUCATION}

\section{Résumé}

Dans cet article, nous dressons des portraits rapides de nos pratiques de conseillers pédagogiques (CP) et abordons quelques-unes des perspectives et valeurs qui nous guident dans notre accompagnement des enseignants au primaire, au secondaire et au collégial. Également, nous revenons sur deux recherches collaboratives que nous avons initiées et auxquelles nous avons activement pris part afin d'en tirer quelques enseignements. Cet examen nous permet de mettre en évidence que nous sommes des CP collaboratifs informés, entre autres, par la recherche dans notre travail d'accompagnement. Également, nous soulignons le fait que nos expériences de recherche nous permettent d'affiner nos expertises du processus d'accompagnement.

Mots-clés : conseiller pédagogique, réflexion, pratique, accompagnement, processus 


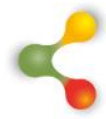

\section{REVUE HYBRIDE DE L'ÉDUCATION}

\section{Mise en contexte}

Comme conseillers pédagogiques (CP), nous possédons, chacun, une décennie d'expériences d'enseignement au primaire (treize ans pour Mme Murray) et au secondaire (douze ans pour M. Perron). Nous sommes des praticiens aguerris qui avons décidé à un moment donné de nos parcours professionnels de nous mettre au service de l'accompagnement des enseignants, et ce, à titre de CP, voire même de superviseure de stage (Murray). Une des choses qui nous distinguent, entre autres, est notre capacité à accompagner les enseignants de deux ordres d'enseignement différents de ceux où nous avons pratiqué, soit le primaire (pour M. Perron) et le collégial (pour Mme Murray). Une des clefs de nos succès respectifs comme CP est certainement nos perspectives ou nos valeurs qui apportent un certain éclairage sur les pratiques enseignantes et transcendent les niveaux d'enseignement. Dans cette première section, nous déclinons quelques-unes de ces perspectives et valeurs.

\subsection{Une perspective systémique d'amélioration des pratiques}

Un des avantages d'accompagner à la fois les enseignants plus généralistes du primaire et les spécialistes du secondaire, est d'éviter de tomber dans une sorte de surspécialisation. On est ainsi amené à ne pas se limiter au niveau micro des disciplines à enseigner (par exemple les mathématiques ou les sciences) et à considérer le niveau macro, ce qui permet de voir plus grand, d'essayer en quelque sorte de jouer sur la grande toile. Ainsi, nous pouvons identifier de beaux dossiers tels la rétroaction par les élèves en cours d'apprentissage, l'évaluation formative, l'enseignement réciproque, la résolution de problèmes, la motivation, l'intégration des technologies, etc. Avec ces dossiers, autour de thématiques, de préoccupations ou d'approches transversales, nous pouvons espérer des retombées plus grandes sur le système, par exemple en contribuant à déployer des pratiques gagnantes non seulement pour une classe ou un niveau, mais également pour des milliers d'élèves et d'enseignants. Au collégial, l'approche-programme prônée par la réforme a accentué le caractère collectif du travail des enseignants qui doivent travailler davantage en collaboration pour favoriser la réussite des étudiants. L'impact de cette réforme et de l'approche par compétences a eu des effets sur le rôle du CP au collégial qui devient un expert des stratégies pédagogiques qui accompagne les enseignants dans leurs projets pédagogiques particuliers ou en équipes disciplinaires relevant de différents programmes. Le CP est une ressource essentielle au service des programmes pour des projets pédagogiques portés par l'équipe des divers professionnels et enseignants ou sur la base des éléments plutôt administratifs mis en évidence par les politiques où les coordonnateurs ${ }^{1}$

\footnotetext{
${ }^{1}$ Les coordonnateurs sont des enseignants bénéficiant de dégagements d'enseignement afin de s'occuper des opérations normales pour les départements : faire des réunions, planifier les tâches et
} 


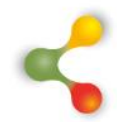

\section{REVUE HYBRIDE DE L'ÉDUCATION}

avec le CP doivent travailler en étroite collaboration dans leurs régulières redditions de compte. À cet égard, l'obligation de rendre des comptes (qui résulte d'une plus grande autonomie des collèges) vient ajouter de nouveaux dossiers au plan de travail des $\mathrm{CP}$, de nouveaux dossiers qui risquent d'augmenter l'aspect administratif de la fonction de CP au détriment de l'aspect pédagogique (Houle et Pratte; 2007). Cet aspect pédagogique s'illustre, entre autres, à travers le rôle que le CP est amené à jouer suite aux évaluations annuelles qu'il analyse avec les coordonnateurs (statistiques à l'appui, par exemple taux de réussite, taux de diplomation, taux de réinscription, etc.) pour cibler avec les équipes enseignantes les meilleures pistes d'action possibles telles que des formations ou des ateliers sur les stratégies pédagogiques à favoriser.

\subsection{Améliorer les pratiques par l'accompagnement réflexif}

CEuvrer à améliorer les pratiques enseignantes par divers moyens d'accompagnement est un des créneaux de la fonction d'un CP. Un de ces moyens est d'amener les enseignants à faire une réflexion ou à pratiquer l'introspection sur leurs propres pratiques, à réfléchir sur celles qui semblent les plus efficaces ou qui favorisent la réussite du plus grand nombre d'élèves. Notre privilège comme CP est de pouvoir influencer la réussite de milliers d'élèves à travers les pratiques de plusieurs enseignants alors qu'avant, à titre d'enseignants, nous ne pouvions influencer que les élèves que nous côtoyions. L'accompagnement réflexif nous a amenés, au fil des ans, à développer différents dispositifs, diverses approches pour faire cheminer les enseignants. Par exemple, le recours au journal de bord élaboré dans le cadre d'une étude que nous (Murray) avons menée au Cégep de Jonquière (voir figure 1). Cet outil propose des pistes de réflexion pour accompagner individuellement les enseignants du collégial dans l'analyse de leurs pratiques pédagogiques, et ce, en lien avec un profil des compétences professionnelles au collégial développé exclusivement dans le cadre de cette étude menée en collaboration avec différents intervenants (enseignants représentant différents secteurs, syndicat local, coordonnateurs et conseillers pédagogiques).

\subsection{Un accompagnement pragmatique et valorisant}

À titre de $\mathrm{CP}$, il s'agit donc non seulement de faire réfléchir les enseignants sur les pratiques efficaces, que celles-ci émanent ou non de leurs expériences, mais surtout de se questionner sur comment consolider ces pratiques dans les classes. On ne parle pas ici de lectures que l'on tente d'appliquer, mais d'une approche pragmatique et synergique qui renvoie au comment faire pour mettre en forme les pratiques prometteuses, les approches porteuses. Ce souci du concret nous a conduits, à la Commission scolaire De la Jonquière, à envisager des voies et moyens

horaires des enseignants, faire les remplacements d'enseignants, faire des papiers administratifs, faire les budgets, gérer les budgets des équipements. 


\section{6}

\section{REVUE HYBRIDE DE L'ÉDUCATION}

pour faire vivre aux enseignants une approche comme celle de " la rétroaction par les élèves ». Ainsi, ces derniers, dans plusieurs classes, votent à la fin d'un enseignement explicite, à l'aide des verres de couleurs ou des bâtons ou encore avec les pouces. Ils peuvent alors signifier " j'ai compris » ou « je n'ai pas compris » et ainsi permettre à l'enseignant d'avoir une rétroaction immédiate sur son enseignement et, si nécessaire, il pourra diviser ses groupes en fonction de leurs besoins pour ajuster ses stratégies pédagogiques. Dans le même ordre d'idées, une autre manière concrète que nous avons élaborée pour opérationnaliser l'évaluation formative, c'est de laisser aux élèves le choix du type d'examen: des examens de type " étoile » ou de type " défis ", le format révélant alors plus rapidement les matières où les élèves ont plus ou moins de difficultés. Ce côté opérationnel de l'accompagnement est gagnant en guidant la discussion pour éviter de trop élargir ou de s'écarter du sujet principal. Par ailleurs, toujours par souci d'être pragmatiques dans l'accompagnement, nous nous gardons de "pelleter de la gravelle » (Perron), c'est-à-dire d'être tout le temps avec les enseignants à essayer plein de petites choses qui, au final, nous éparpillent, nous conduisent à la fatigue et ne font pas beaucoup progresser. Plutôt que de "pelleter de la gravelle ", il est plus utile de choisir deux ou trois grosses « roches " et de les pousser à fond, de les travailler plus profondément avec les enseignants. Egalement, comme CP, il est important d'accepter le savoir-faire et le savoir d'expérience des enseignants et de ne pas vouloir inculquer sa manière de faire comme expert. Une telle attitude est valorisante pour les enseignants qui sont dans la partie opérationnelle du quotidien et qui transigent avec les imprévus et les caractéristiques des élèves. Le CP a comme rôle de miser sur les forces des uns et des autres, de réunir les enseignants dans des communautés de pratiques où la place est à la discussion dans le respect de ce qui fonctionne, où on met en commun les choses qui fonctionnent; où on rend les choses concrètes, cohérentes et compréhensibles. Ce genre de collaboration nous a permis, au secondaire, de proposer un outil d'enseignement efficace que nous continuons de peaufiner avec l'intention de l'utiliser également au primaire. Cet outil est une carte conceptuelle décisionnelle (voir figure 2). La carte conceptuelle permet à l'enseignant de présenter un chapitre de volume en 30 minutes puis de faire progresser ses élèves à travers des tâches complexes. Les élèves, pour employer une analogie, "battent des sentiers » à travers la carte, « se promènent » dans la carte, organisent leurs choses au moyen de la carte qui leur permet de faire des liens et leur fournit un plan d'action. C'est alors une approche différente et une belle façon d'en finir avec les enseignements longs et magistraux que les élèves ne suivent pas. 


\section{REVUE HYBRIDE DE L'ÉDUCATION}

Uutil de développement professionnel

Enseignement collégial

\section{PISTES DE RÉFLEXTON}

Cours : _ Session :

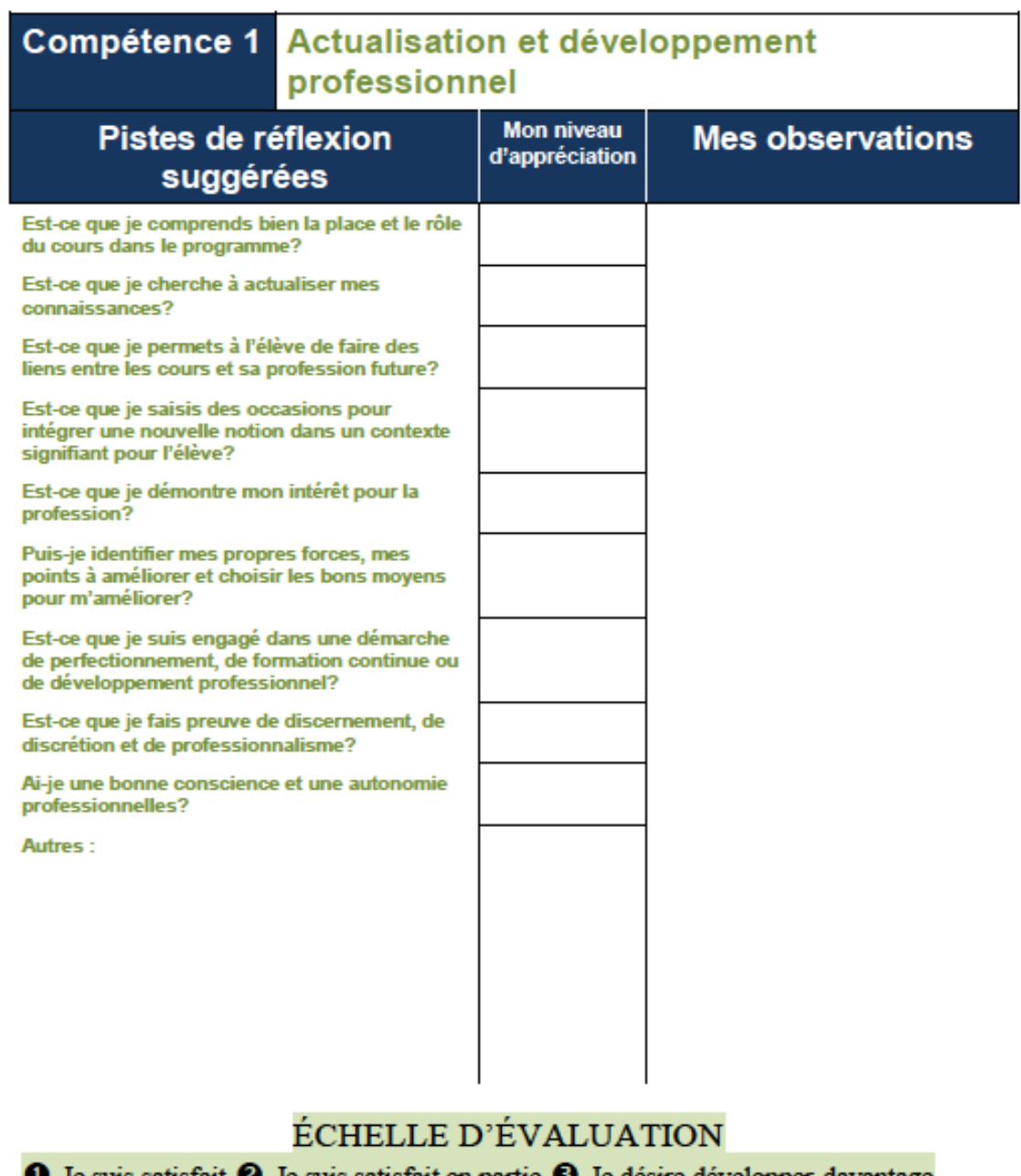

(1) Je suis satisfait 2 Je suis satisfait en partie 3 Je désire développer davantage Senice d' cunimation at de developpement padiagogigues - Cogep de Jonquiere

Figure 1 : Journal de bord réflexif (Murray, 2012) 


\section{$\&$}

\section{REVUE HYBRIDE DE L'ÉDUCATION}

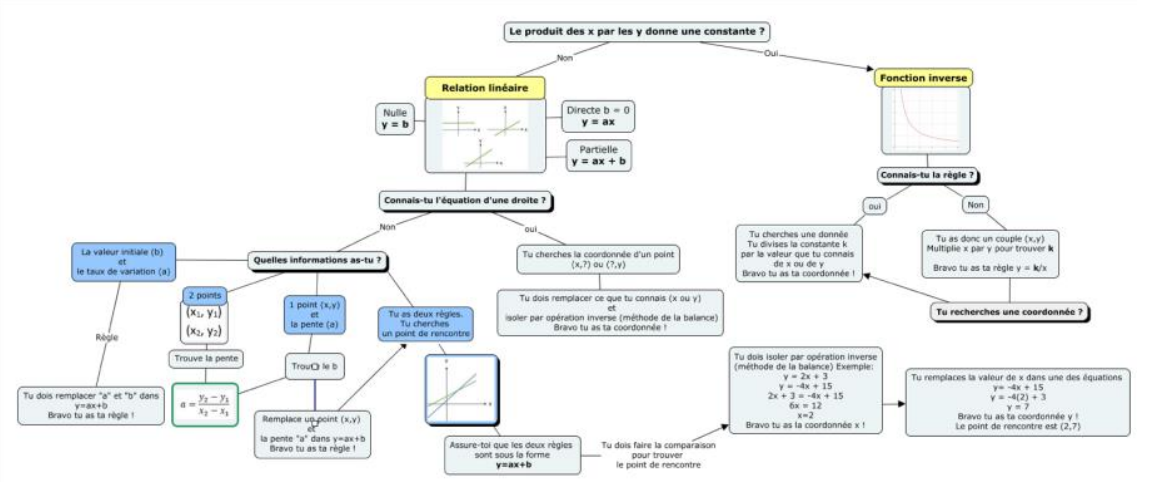

Figure 2 : Les fonctions de $3^{\mathrm{e}}$ secondaire : une carte conceptuelle pour te guider (Étienne Perron et Yan Bilodeau)

\section{Un travail de CP également informé par la recherche}

Sans nul doute, la recherche nourrit nos pratiques de CP dans la mesure où nous nous servons à différentes fins de résultats intéressants issus de travaux de recherches que ce soit pour appuyer nos propos, pour élargir nos perspectives, pour aider à documenter les formations que nous donnons ou encore pour rester alertes face aux nouveautés. La veille scientifique est une activité fondamentale qui guide nos interventions afin d'être au diapason des innovations pédagogiques tant au primaire qu'au secondaire et au collégial. Également, nous voyons la recherche comme une occasion ou un prétexte pour expérimenter ou implanter des pratiques et de nouveaux outils pédagogiques jugés à fort potentiel. À cet égard, mentionnons par exemple les recherches sur l'enseignement réciproque dont le facteur élevé d'influence a été établi à 0.73 par Hattie (2009). Nous continuons donc, dans notre (Perron) accompagnement des enseignants, d'explorer les possibilités qu'offre l'enseignement réciproque. Dans un cas (Perron), pour ce qui est de notre rapport à la recherche, nous pourrions parler d'une perspective pragmatique de la recherche et qui exige une grande ouverture à des approches différentes et complémentaires. Dans un autre cas (Murray), notre rapport à la recherche est le même pour Perron. Cependant, nous avons développé une particularité : nous avons eu à nous impliquer dans de nombreuses recherches dédiées (coaching pédagogique ; développement professionnel ; motivation dans les cours de mathématiques; pédagogie interculturelle avec les étudiants internationaux; troubles de fatigue, anxiété, chez les étudiants en soins infirmiers), sans oublier les années de formation à compléter une recherche doctorale terminée en 2014 (Murray, 2014). Dans le cas particulier de Murray, nous pouvons parler d'un intérêt marqué pour la recherche qui alimente et structure l'accompagnement, un accompagnement marqué par le coaching qui a été influencé par le domaine de la gestion dans une perspective d'évaluation, d'entrer en relation, de conseiller, de proposer des défis afin que l'enseignant veuille s'impliquer dans son développement 


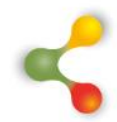

\section{REVUE HYBRIDE DE L'ÉDUCATION}

professionnel. C'est un accompagnement qui demande de faire un suivi régulier suite aux échanges ou aux entretiens, aux expérimentations faites ou aux observations que nous avons pu faire en classe. Diverses actions, nous (Murray) ont amenés à jouer un rôle de coach pédagogique confirmant l'autre dans ce qu'il devient, ou en agissant comme un miroir pour lui, le tout dans un climat de confiance, de confidentialité et de sécurité avec un souci de croissance et de bien-être de l'autre.

\subsection{Une recherche collaborative au secondaire sur l'intégration du TNI dans l'enseignement des mathématiques}

Entre 2013 et 2016, nous (Perron) avons pris part à une recherche collaborative que nous avons initiée et qui impliquait des enseignants de mathématiques au secondaire que nous accompagnions auparavant (Barry et Perron, 2014; Barry, Ouellet et Perron, 2015). La recherche portait sur l'intégration du TNI dans l'enseignement des mathématiques en classe de secondaire 5. Durant cette étude, CP, chercheur et enseignants se sont entendus pour comparer, à différents moments et autour des mêmes contenus d'enseignement (graphes et chaines, transformations géométriques, fonctions trigonométriques) une classe n'utilisant pas le TNI et une autre intégrant le TNI. Une telle comparaison nous a permis de questionner ensemble la valeur ajoutée supposée des TIC en termes des apprentissages faits par les élèves ainsi que des pratiques mobilisées par les enseignants. Également, cette recherche a été une occasion de croiser les perspectives des uns et des autres et de susciter des échanges féconds sur le passage aux TIC pour la classe traditionnelle ainsi que sur l'intégration, voire la meilleure intégration, des TIC pour la classe TIC. À titre de $\mathrm{CP}$, un des enseignements importants que nous tirons de cette recherche collaborative est en lien avec l'accompagnement gagnant à offrir aux enseignants TIC afin de les sensibiliser davantage aux risques d'appauvrissement à la fois de l'activité mathématique et du TNI en dépit des nombreuses opportunités d'enrichissement qu'ils présentent, certaines de ces opportunités étant documentées par cette étude.

\subsection{Une recherche collaborative au collégial pour accroitre la motivation des étudiants dans les cours de mathématiques}

Entre 2016 et 2018, nous (Murray) avons initié et mené une recherche collaborative en collaboration avec des enseignantes de mathématiques au collégial intéressées par la question de la motivation des étudiants dans le cadre des cours qu'elles dispensaient. Dans cette étude, il avait été convenu d'aborder la question de la motivation en s'attardant aux « pratiques effectives ", tout en fournissant l'opportunité aux enseignantes de revisiter et de questionner leurs « pratiques existantes " qui, en partie, ont été documentées sous forme d'enregistrements vidéo pour être ensuite analysées. Dans cette recherche collaborative, notre expérience en recherche nous a permis de jouer un rôle central et inusité à titre de CP. Ainsi, en plus de coordonner la logistique de la recherche 


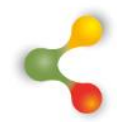

\section{REVUE HYBRIDE DE L'ÉDUCATION}

dans le milieu de travail, nous avons été cochercheures en acceptant d'élaborer conjointement les canevas d'entrevues, mais surtout en conduisant nous-mêmes presque toutes les entrevues individuelles d'autoconfrontation simple. Lors des entrevues individuelles, qui faisaient suite aux observations en classe, chaque enseignante observée a été confrontée aux vidéos de son activité en classe, et ce, pour expliciter des aspects de sa pratique pertinents pour ce qui est du soutien apporté à la motivation et à la persévérance en mathématiques des étudiants. Ces entrevues et les rôles que nous avons été amenés à jouer comme CP illustrent bien la fonction pédagogique de l'accompagnement (Le Boterf, 1999), soit aider la personne accompagnée à nommer ce qu'elle fait et à identifier les problèmes qu'elle rencontre dans sa pratique, la mettre en relation avec des ressources et des connaissances pertinentes aux problèmes rencontrés (ce que St-Arnaud [1999] appelle la fonction de suppléance) et, finalement, l'aider à faire le point sur sa démarche et sa progression. Comme $\mathrm{CP}$, nous avons également pris une part active à l'orchestration de chaque autoconfrontation croisée. Cette approche constitue la pierre d'assise d'un travail collaboratif impliquant des enseignants expérimentés, le chercheur (expert de la discipline et du processus) et la CP (experte en pédagogie et accompagnement). L'idée, avec l'autoconfrontation croisée, est d'apprendre sur sa propre pratique en discutant avec le groupe pour cheminer dans la compréhension et l'amélioration effective de sa pratique (Payette, 2009). II s'agissait d'adopter une posture de " praticien réflexif " à partir d'une pratique congruente d'autoanalyse de ses interventions professionnelles en vue de les améliorer. Bien que le travail individuel réflexif demeure nécessaire et bien qu'il puisse être alimenté par des activités de formation telles que des lectures personnelles, des cours, des conférences, etc., nous croyons que le partage d'expériences, la réflexion collective ainsi que des discussions et des échanges structurés suscitent une dynamique d'interaction professionnelle qui est une puissante source de motivation pour atteindre les objectifs d'une recherche.

\section{Apports et perspectives de l'implication dans les recherches collaboratives}

La recherche collaborative sur l'intégration du TNI dans l'enseignement des mathématiques en classe de secondaire nous (Perron) a davantage fait réaliser l'importance, dans des études ultérieures du même genre, de former explicitement certains enseignants à quelques usages des technologies. Même si cela a été fort pertinent de partir du niveau effectif d'intégration du TNI des enseignants prenant part à la recherche et malgré le fait qu'ils ont beaucoup appris des échanges riches et constructifs entre participants lors des rencontres réflexives de bilan que nous coanimions, il aurait été possible d'améliorer les compétences TIC des enseignants qui auraient plus évolué ou cheminé durant la réalisation du projet. Ceci soulève tout de même une question sur le rôle du CP dans ce type de recherche, où le questionnement de pratique pourrait davantage 


\section{$\&$ \\ REVUE HYBRIDE DE L'ÉDUCATION}

être soutenu par une formation plus explicite, à l'interne, ou prise en charge principalement par le CP. Cependant, ce rôle du CP doit être négocié avec le chercheur. Des formations ciblées, à l'interne, à des moments choisis afin de répondre aux besoins constatés ou exprimés ou encore aux besoins de mise à niveau pour améliorer certaines pratiques, sans oublier de documenter ces améliorations durant la recherche. Par ailleurs, une fois la recherche complétée, certains de nos résultats obtenus, sous toutes réserves, auraient dû être plus diffusés. À titre d'exemple de résultats qui auraient dû faire l'objet d'une plus grande diffusion dans les milieux de pratiques, on retrouve la comparaison entre classe dite traditionnelle (sans TNI) et classe intégrant le TNI qui a mis en évidence des sortes de « boites noires ». Grâce au TNI, les élèves peuvent s'émerveiller de certaines manipulations qui, pour ainsi dire, volent la vedette aux processus plus fondamentaux et qui sous-tendent ces manipulations. Hélas, comme nous avons eu à le constater, ces processus ont été mieux valorisés dans la classe traditionnelle où les élèves ont fait des apprentissages importants que leurs collègues de la classe TNI et confrontés aux mêmes contenus n'ont pas fait. Assurément, le TNI doit être à la disposition de la classe et de l'enseignant, mais les élèves doivent également pouvoir l'utiliser, au risque de tomber dans une sorte de "one-man show » de l'enseignant qui impressionne! II s'agit donc de souligner davantage certains résultats intéressants obtenus, certaines retombées. Au nombre de ces retombées, on ajoute l'expérience riche de la démarche de recherche, le processus dans lequel les participants se sont engagés tout au long de l'étude.

Quant à la recherche collaborative portant sur la motivation des cégépiens dans les cours de mathématiques, elle a permis, entre autres, aux enseignantes participantes de questionner leurs pratiques, de valider les approches et les outils mis en place au fil des années. Ainsi, selon le cas, elles se sont vues confortées dans certaines de leurs pratiques et encouragées à ne pas poursuivre avec d'autres pratiques moins propices à accroitre la motivation des étudiants dans les cours de mathématiques (voir Barry, Saboya et Murray, dans ce numéro thématique). Sans doute pour les enseignantes, comme dans d'autres recherches participatives, elles se sont développées professionnellement et ont amélioré leurs pratiques. Une fois la recherche terminée, les quelques échos reçus des suites données par les enseignantes participant à la recherche sont une plus grande ouverture aux résultats de recherches ainsi qu'aux expérimentations en classe de diverses approches porteuses. Nous (Murray) avons apprécié la flexibilité de la démarche qui nous a obligés, $\mathrm{CP}$ et chercheur, à bâtir de façon émergente les canevas ayant servi aux différentes autoconfrontations et que nous expérimentions pour la première fois. Cette exploration nous permet donc, à l'avenir, de nous doter de guides d'entrevues mieux élaborés à la lumière des enseignements tirés de l'étude réalisée. Les résultats diffusés de cette recherche ont mis en lumière la méthodologie de celle-ci. Nous avons insisté sur ce point dans les communications que nous avons eu à faire à propos de cette recherche. Pour nous, les aller-retours entre autoconfrontation simple (en individuel) 


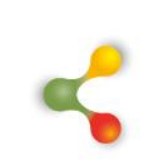

\section{REVUE HYBRIDE DE L'ÉDUCATION}

et autoconfrontation croisée (en collectif) ont été fort enrichissants pour accompagner les enseignantes et pour documenter les effets de cet accompagnement. Sans doute, l'expérience des autoconfrontations simples permet de bonifier notre approche pédagogique du coaching, sans oublier l'expérience des autoconfrontations croisées qui pourrait aider les $\mathrm{CP}$ à inventer de nouvelles formes d'accompagnement collectif. D'ailleurs, cette recherche a permis de constater un manque d'outils pour les CP euxmêmes. Ceux-ci doivent pouvoir être guidés dans l'analyse de leurs pratiques d'accompagnement. Nous avons élaboré un outil : le journal de bord pour le conseiller pédagogique (voir figures 3 et 4). II a été élaboré sous la forme de celui expérimenté par les enseignants. On y retrouve un portrait détaillé et abrégé du profil de compétences d'un CP défini dans la recherche menée par Houle et Pratte (2007) suivi d'une section de pistes de réflexion par compétence ciblée. Le CP peut alors approfondir sa réflexion en fonction de chacune des compétences de son profil et se fixer des objectifs réalistes en plus de cibler des moyens nécessaires à mettre en place pour s'améliorer et poursuivre son développement professionnel (Murray, 2017). 


\section{REVUE HYBRIDE DE L'ÉDUCATION}

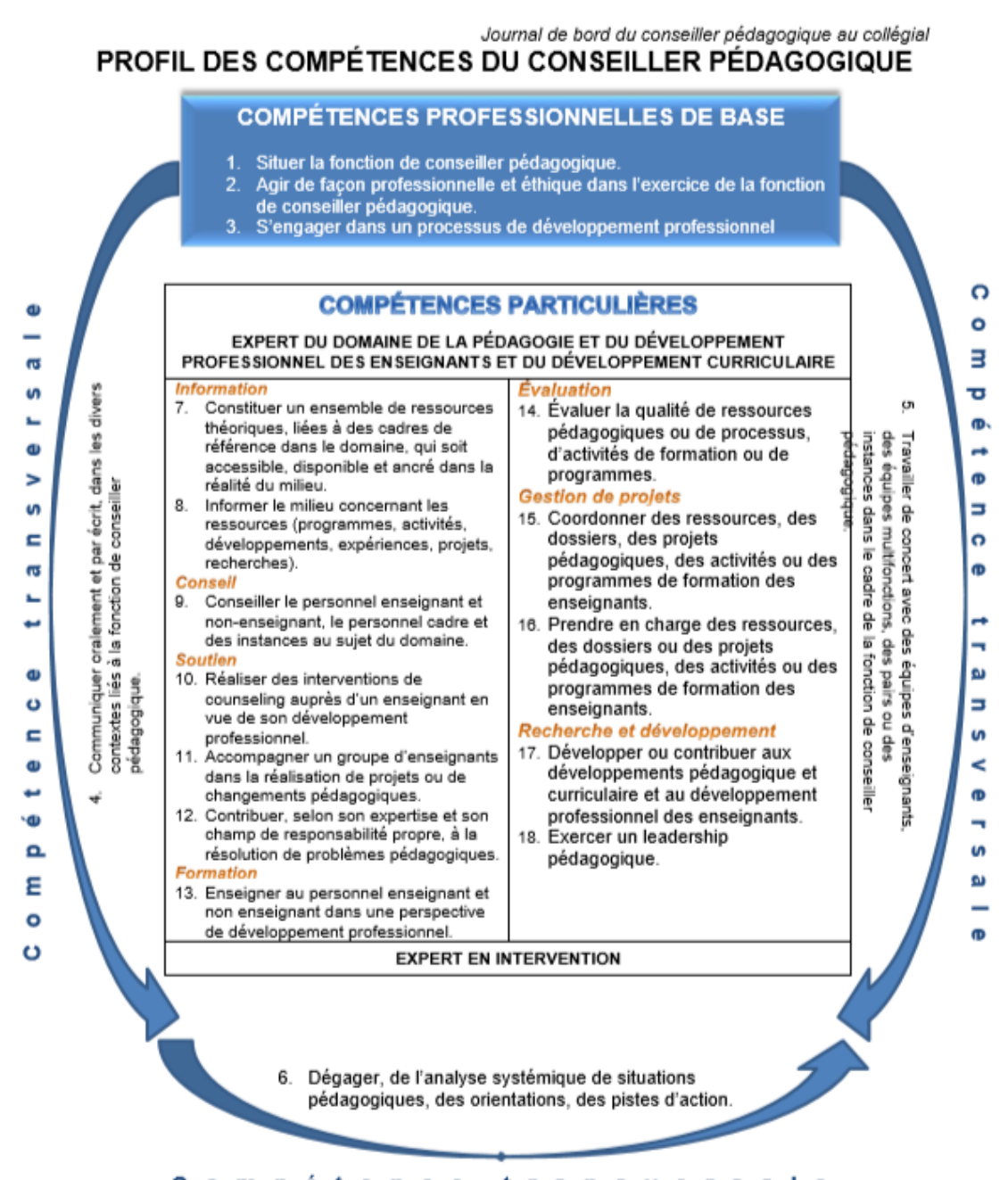

C o m pé t e n c e tra n s vers a l e

Figure 3 : Profil des compétences professionnelles du CP (Murray, 2017) 


\section{REVUE HYBRIDE DE L'ÉDUCATION}

Journal de bord du conseiller pédagogique au collégial

\section{PISTES DE RÉFLEXION}

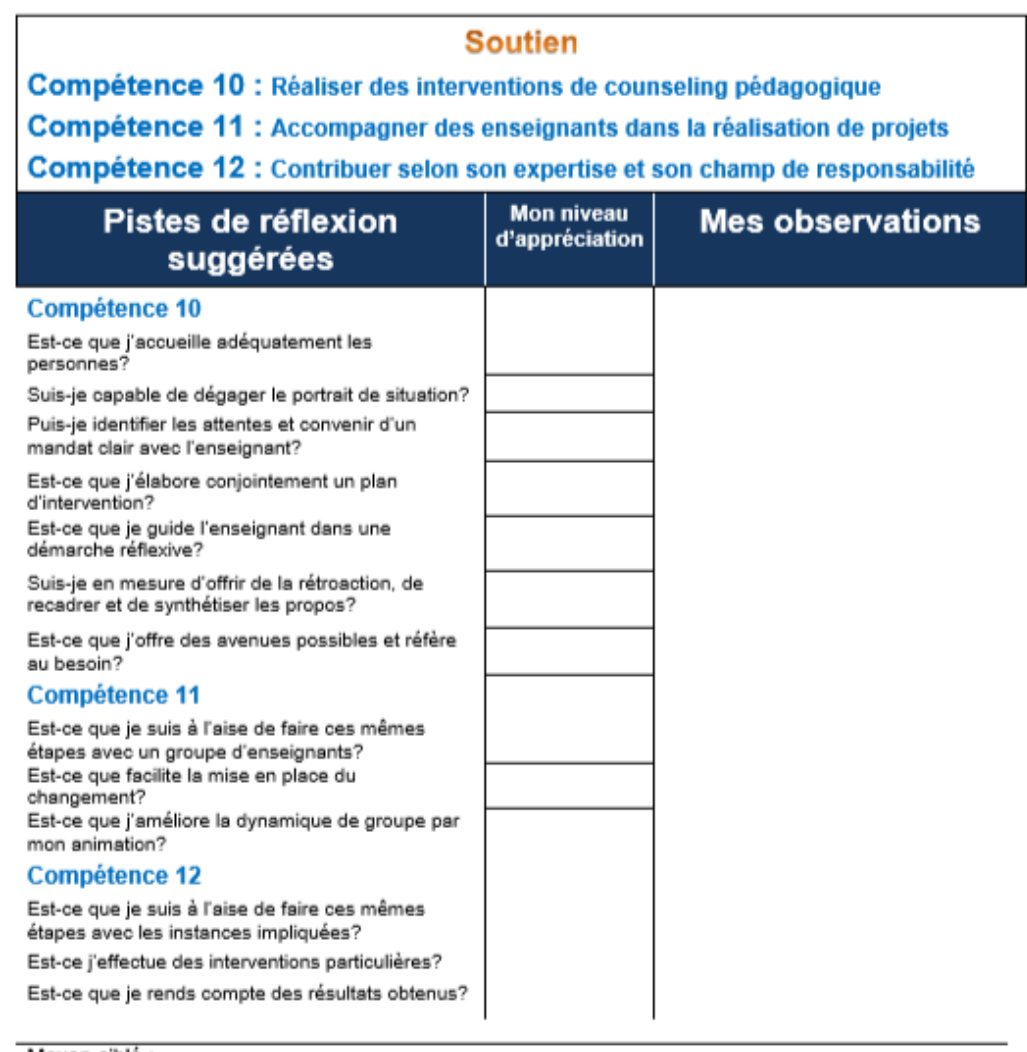

Moyen ciblé

\section{ÉCHELLE D'ÉVALUATION}

( Je suis satisfait 2 Je suis satisfait en partie 3 Je désire développer davantage Service d'animation et de développement pédagogiques - Cégep de Jonquière Page 30

Figure 4 : Pistes de réflexion (Murray, 2017)

\section{Conclusion}

Pour conclure, à titre de $\mathrm{CP}$, nous pouvons témoigner d'une autre retombée importante de notre participation à une recherche collaborative, celle d'affiner notre expertise du processus d'accompagnement. En l'occurrence, à la lumière des autoconfrontations simples (entrevues individuelles) et des coanalyses de groupe, il y a beaucoup à apprendre à 


\section{$\&$}

\section{REVUE HYBRIDE DE L'ÉDUCATION}

propos du questionnement de pratique (qui ne va pas de soi, ni pour les enseignants ni pour les CP) ainsi que sur la conduite des rencontres réflexives. Nous (Murray) avons davantage pris conscience de l'importance de la relation dans tout accompagnement, et surtout, du volet affectif dans le questionnement de pratique. À cet égard la collaboration entre CP (davantage expert des processus) et chercheur-didacticien (davantage expert des contenus) permet d'optimiser l'accompagnement du questionnement des praticiens en recherche collaborative, en misant sur leur complémentarité. C'est assurément là une formule gagnante pour d'autres recherches collaboratives au collégial. Enfin, un gros investissement est requis des participants dans une recherche collaborative. Ce type d'implication est plus exigeant que dans bien d'autres recherches. Pour soutenir cet investissement, il faut trouver les moyens de libérer les $\mathrm{CP}$ et les enseignants et croire au potentiel de la recherche collaborative. Enseignants et $\mathrm{CP}$, dans notre recherche (Murray), ont dû sacrifier beaucoup de leur temps personnel, à la fin des cours ou entre des cours, pour prendre activement part aux différentes rencontres. Les cégeps, surtout, mais également les commissions scolaires, devraient permettre ces libérations pour les participants d'une recherche dans les financements qu'ils accordent, des libérations supplémentaires s'ajoutant aux subventions, que certaines administrations ne considèrent pas encore. Un temps suffisant (demi-journée ou journée entière) doit être dégagé à l'horaire pour permettre une implication de plus grande qualité des participants aux recherches collaboratives. 


\section{REVUE HYBRIDE DE L'ÉDUCATION}

\section{Références}

Barry, S. et Perron, E. (30 avril, 2014). De la classe TIC[E] à la classe $T I C[C]$ : perspectives croisées [communication orale]. Consortium Régional de Recherche en Éducation. Chicoutimi, QC, Canada.

Barry, S., Ouellet, K. et Perron, E. (2015). Un outil populaire, puissant, mais encore. Repéré à : http://rire.ctreq.qc.ca/2015/10/tni-outil/.

Hattie, J. (2009). Visible learning : a synthesis of over 800 meta-analyses relating to achievement. Routledge.

Houle, H. et Pratte, M. (2007). La fonction du conseiller pédagogique au collégial: rapport de recherche. Université de Sherbrooke. https://www.usherbrooke.ca/performa/fileadmin/sites/performa/do cuments/Recherches subventionnees/Houle et Pratte Final An nexes.pdf

Le Boterf, G. (1999). De l'ingénierie de la formation à l'ingénierie des compétences. Dans P. Carré et Caspar, P. (Dir.), Traité des sciences et des techniques de la formation (p.335-353). Dunod.

Murray, N. (2012). Étude sur les activités de développement professionnel chez les enseignants au collégial. Activités de formation continue à privilégier. Cégep de Jonquière.

Murray, N. (2012). Développement professionnel. Journal de bord. Outil de réflexion. Cégep de Jonquière.

Murray, N. (2014). Portrait des activités privilégiées par les enseignants en exercice pour le développement des compétences professionnelles en contextes préscolaire, primaire et secondaire [Thèse de Doctorat inédite]. Université du Québec à Chicoutimi.

Murray, N. (2017). Développement professionnel du conseiller pédagogique. Journal de bord. Outil de réflexion. Cégep de Jonquière.

Payette, P. (1993). L'efficacité des gestionnaires et des organisations. Presses de l'Université du Québec.

Payette, A. (2009). Le groupe de codéveloppement et d'action formation. Une approche puissante encore méconnue. Dans D. Bouteiller et L. Morin. Développer les compétences au travail (p.439-451). HEC. 


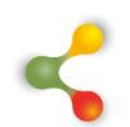

\section{REVUE HYBRIDE DE L'ÉDUCATION}

St-Arnaud, Y. (1999). Le changement assisté - Compétences pour intervenir en relations humaines. Gaëtan Morin éditeur. 